\title{
ChemComm
}

Cite this: Chem. Commun., 2014 50,1131

Received 7th October 2013,

Accepted 30th October 2013

DOI: $10.1039 / \mathrm{c3cc47696k}$

www.rsc.org/chemcomm

\section{Stereoselective synthesis of folded luminogens with arene-arene stacking interactions and aggregation-enhanced emission $\dagger$}

\author{
Zujin Zhao, ${ }^{\mathrm{ab}}$ Bairong He, ${ }^{\mathrm{b}}$ Han Nie, ${ }^{\mathrm{a}}$ Bin Chen, ${ }^{\mathrm{b}}$ Ping Lu, ${ }^{\mathrm{c}}$ Anjun Qin ${ }^{\mathrm{a}}$ and \\ Ben Zhong Tang*ad
}

\begin{abstract}
Tetraphenylethene derivatives with a folded Z-conformation and aggregation-enhanced emission characteristics are synthesized from 2-arylbenzophenones. The intramolecular rotation of the folded luminogens is partially suppressed, resulting in improved emission efficiencies in solutions.
\end{abstract}

Since the discovery of aggregation-induced emission (AIE) by Tang et al. in 2001, ${ }^{1}$ the design and synthesis of new AIE luminogens and exploration of their potential applications have attracted rapidly increasing interest. The novel AIE phenomenon is the direct opposite of aggregation-caused quenching (ACQ), which is well-known and widely observed from conventional chromophores. Delineating the mechanism of the AIE phenomenon is essential not only for fundamental understanding, but also, for advancing various new AIE systems. For propeller-like luminogens, e.g., tetraphenylethene (TPE), the restriction of intramolecular rotation (RIR) is hypothesized to be the main reason underlying their AIE effects. ${ }^{2,3}$ While many indirect experimental and computational results support the RIR hypothesis, direct evidence from intramolecular control of the rotations is limited. Most subtle structural alterations to achieve RIR involve chemical bonding between aromatic rotors, which may alter the chemical nature, and thus the optical properties of the luminogens to a certain extent. ${ }^{4}$

\footnotetext{
${ }^{a}$ State Key Laboratory of Luminescent Materials and Devices,

South China University of Technology, Guangzhou 510640, China.

E-mail: zujinzhao@gmail.com, tangbenz@ust.hk

${ }^{b}$ College of Material, Chemistry and Chemical Engineering,

Hangzhou Normal University, Hangzhou 310036, China

${ }^{c}$ State Key Laboratory of Supramolecular Structure and Materials, Jilin University, Changchun 130012, China

${ }^{d}$ Department of Chemistry, Institute of Molecular Functional Materials, The Hong Kong University of Science \& Technology, Clear Water Bay, Kowloon, Hong Kong, China

$\dagger$ Electronic supplementary information (ESI) available: Experimental details, X-ray crystallography, ${ }^{1} \mathrm{H}$ NMR spectra, proposed mechanism of the reaction, crystal structure of compound 10, and Table S1. CCDC 955718-955721. For ESI and crystallographic data in CIF or other electronic formats see DOI: 10.1039/ c3cc47696k
}

McMurry coupling is an effective method of building $\mathrm{C}=\mathrm{C}$ double bonds from ketones or aldehydes. ${ }^{5}$ It has been widely applied to the preparation of TPE and its derivatives. ${ }^{3 c, 6}$ The reaction proceeds efficiently and affords stereo-uniform products by adopting symmetrically modified benzophenones as substrates. The products, however, are stereo-random, with nearly equal amounts of $Z-/ E$-isomers, when unsymmetrical benzophenone derivatives are treated under the same conditions, owing to the non-stereoselectivity of the reaction. Recently, we found that McMurry coupling could undergo divergent reaction pathways and furnish diverse products, which were greatly dependent on the nature of the substituents and their positions on benzophenones. ${ }^{7}$ The aromatic group at the 2-position of benzophenone influences the stereoselectivity of the reaction, leading to stereoregular TPE derivatives, which are rarely reported by other groups. ${ }^{8}$ In this work, we wish to focus on the synthesis of folded TPE derivatives and elucidate a mechanism for AIE.

The synthesis of an archetypal folded luminogen ((Z)-o-BPTPE) from 2-phenylbenzophenone (1) is shown in Scheme 1. Three kinds of phenyl rings in (Z)-o-BPTPE are labelled $\phi_{1}, \phi_{2}$ and $\phi_{3}$ for convenient description. The same labelling applies to other folded luminogens as discussed below. The treatment of $\mathbf{1}$ with $\mathrm{TiCl}_{4}$ and zinc dust generates (Z)-o-BPTPE in a moderate yield of $50 \%$. The reaction yields no other TPE derivative apart from (Z)-o-BPTPE. The product is isolated and easily purified by column chromatography. Single crystals of (Z)-o-BPTPE are grown from a THF-ethanol mixture and analyzed by X-ray diffraction crystallography. The crystal structure of (Z)-o-BPTPE illustrated in Fig. 1 confirms that (Z)-o-BPTPE is a $Z$-isomer. It has a folded conformation, in which $\phi_{1}$ and $\phi_{2}$ are located in a nearly parallel manner with the shortest distances of $3.264 \AA$ and $3.274 \AA$ being shorter than the typical distance for $\pi-\pi$ stacking interactions (3.5 $)$ ). The $\phi_{1}$ and $\phi_{2}$ surfaces have an overlap of approximately $50 \%$. This type of parallel, stacked and offset geometry is favorable to the formation of $\pi-\pi$ stacking interactions between $\phi_{1}$ and $\phi_{2} \cdot{ }^{9,10}$ The ${ }^{1} \mathrm{H}$ NMR spectrum of (Z)-o-BPTPE shows distinctive peaks at $\sim 5.7$ ppm (Fig. S1, ESI $\dagger$ ), which are assigned to the $\mathrm{H}_{\mathrm{a}}$ protons. The $\mathrm{H}_{\mathrm{a}}$ in $\phi_{2}$ overlies $\phi_{1}$ with a distance of $3.141 \AA$, or $3.187 \AA$ from $\mathrm{H}_{\mathrm{a}}$ to the center of $\phi_{1}$, indicating that $\mathrm{H}_{\mathrm{a}}$ is located within the 

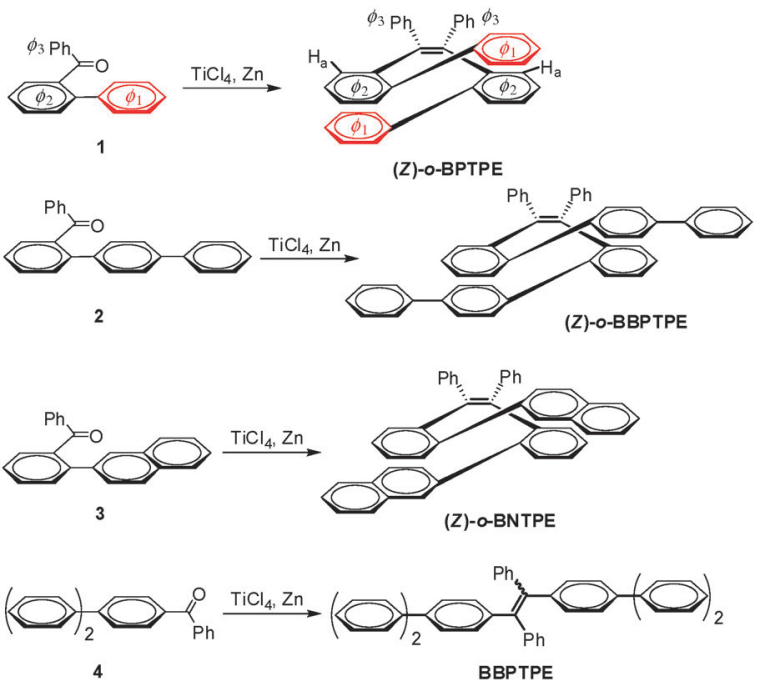

Scheme 1 Syntheses of folded and linear TPE derivatives. The $H_{a}$ protons in (Z)-O-BPTPE are indicated as an example.

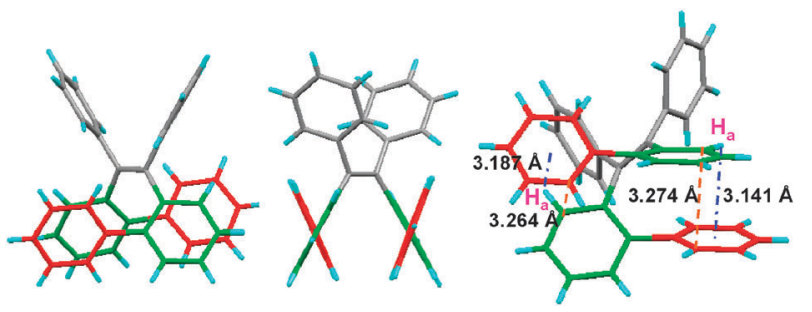

Fig. 1 The X-ray crystallography-determined molecular structure of (Z)-o-BPTPE (CCDC 955718). The shortest distances between $\phi_{1}$ and $\phi_{2}$ and distances from the $\mathrm{H}_{\mathrm{a}} \mathrm{s}$ to the center of $\phi_{1} \mathrm{~s}$ are indicated.

shielding cone of $\phi_{1}$. Thereby, the resonance associated with $\mathrm{H}_{\mathrm{a}}$ moves upfield due to the shielding effect of $\phi_{1}{ }^{11}$

With the successful synthesis of the model molecule (Z)-o-BPTPE, we wondered whether such folded luminogens could be prepared if $\phi_{1} \mathrm{~s}$ were replaced with bulky groups, e.g., biphenyl and naphthalenyl. To our delight, the folded luminogens (Z)-o-BBPTPE and (Z)-o-BNTPE are obtained from 2-biphenyl-4-ylbenzophenone (2) and 2-(naphthalen2 -yl)benzophenone (3) in yields of 56 and $46 \%$, respectively. The distinctive signals from the $\mathrm{H}_{2} \mathrm{~s}$ are recorded at $\sim 5.8 \mathrm{ppm}$ in the ${ }^{1} \mathrm{H}$ NMR spectrum of (Z)-o-BBPTPE (Fig. S2, ESI $\dagger$ ), which are similar to those of (Z)-o-BPTPE. Those of (Z)-o-BNTPE are shifted to a higher field of $\sim 5.3 \mathrm{ppm}$ (Fig. S3, ESI $\dagger$ ), presumably due to the fact that naphthalenyl is richer in $\pi$-electrons and thus, has a stronger shielding effect. However, the reaction of 4-biphenyl-4-ylbenzophenone (4), performed under the same conditions as the control, gives a mixture of $Z$ - $\mid E$-isomers (BBPTPE). The upfield proton resonance signals are absent from the ${ }^{1} \mathrm{H}$ NMR spectrum of BBPTPE (Fig. S8, ESI $\dagger$ ), indicating it has a straight conformation rather than a folded one.

To study the impact of substituent positions on the formation of folded luminogens, 2-phenylbenzophenone derivatives carrying methyl or methoxyl groups at different positions of $\phi_{1}$ are prepared and tested. The reactions of substrates $5 \mathbf{a}$ and $5 \mathbf{b}$, and $\mathbf{6 a}$ and $\mathbf{6 b}$, which possessed methyl or methoxyl groups at the meta- or parapositions of $\phi_{1}$, were carried out successfully to yield single folded

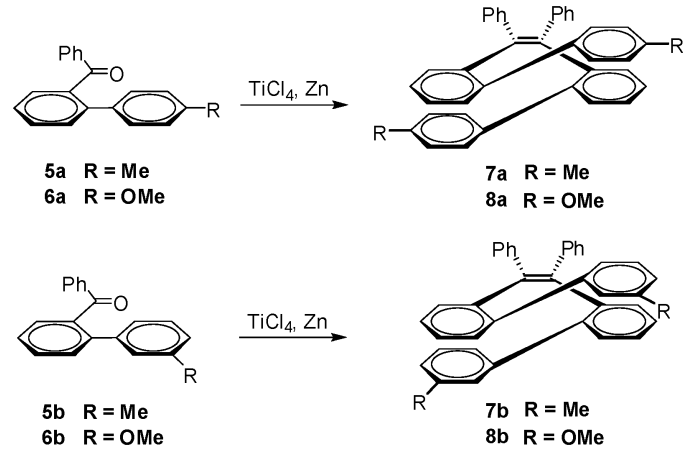

Scheme 2 Syntheses of folded TPE derivatives with methyl or methoxyl substituents at different positions.

TPE derivatives in moderate yields of 40-55\% (Scheme 2). However, the reactions of 2-phenylbenzophenone derivatives with substituents at the ortho-position became complicated and target products were barely isolated. This can be ascribed to the severe steric hindrance of the products. These results reveal that the location of substituents on 2-phenylbenzophenone has a significant impact on the formation of folded luminogens. X-ray diffraction analysis of single crystals of 7a and 8a, grown from THF-ethanol, confirms that both luminogens are $Z$-isomers with a folded conformation (Fig. 2). $\phi_{1}$ and $\phi_{2}$ adopt a cofacial, slightly tilted arrangement with their centers offset, and the shortest distances between them are 3.260-3.339 $\AA$, demonstrating intramolecular $\pi-\pi$ stacking interactions. The $\mathrm{H}_{2} \mathrm{~s}$ in $7 \mathbf{a}$ and 8a are located in a similar position within the crystals as those in (Z)-o-BPTPE. The characteristic chemical shifts of the $\mathrm{H}_{2} \mathrm{~s}$ of $7 \mathbf{a}$ and $7 \mathbf{b}$, and $\mathbf{8 a}$ and $\mathbf{8 b}$, are also recorded upfield at $\sim 5.8 \mathrm{ppm}$ (Fig. S4-S7, ESI $\dagger$ ).

The mechanism behind the formation of the folded $Z$-products was also investigated. Intramolecular $\pi-\pi$ stacking interactions are evidently helpful in stabilizing the intermediate complex of titanium species, the subsequent deoxygenation of which furnishes the folded $Z$-isomers. The coupling of 1-(biphenyl-2-yl)ethanone, an analogue of 2-phenylbenzophenone with a methyl group rather than $\phi_{3}$, only yields an unfolded $E$-product (10, Scheme S1, Fig. S9 and S10, ESI $\dagger$ ) and no folded products. When a methyl group takes the place of $\phi_{3}$,

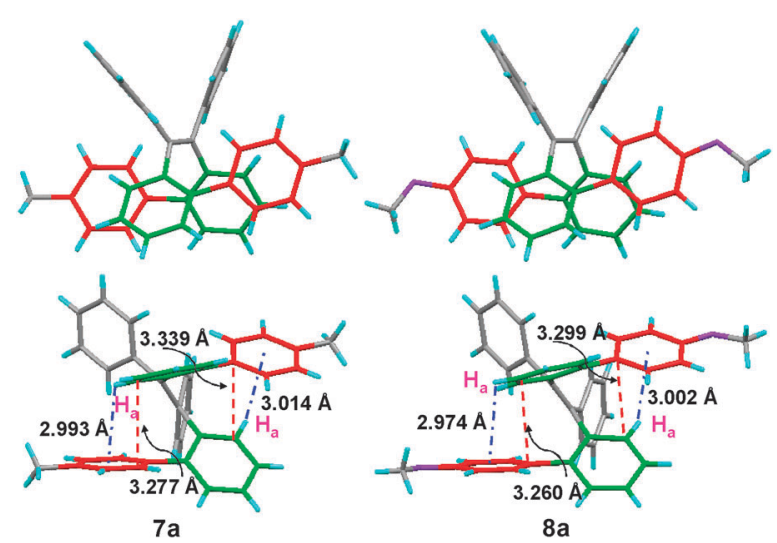

Fig. 2 Crystal structures of 7a (CCDC 955719) and 8a (CCDC 955720). The shortest distances between $\phi_{1}$ and $\phi_{2}$ and distances from the $\mathrm{H}_{\mathrm{a}} \mathrm{s}$ to the center of the $\phi_{1}$ s are indicated. 

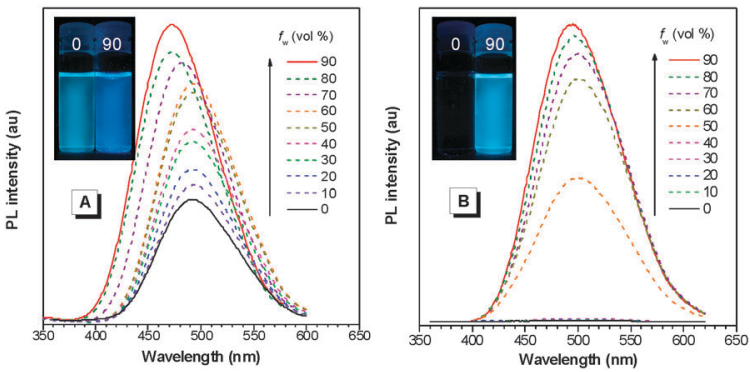

Fig. 3 PL spectra of (A) (Z)-O-BBPTPE and (B) BBPTPE in THF-water mixtures with different water fractions $\left(f_{w}\right)$. Inset: Photo of $(A)(Z)-O-$ BBPTPE and (B) BBPTPE in THF-water mixtures $\left(f_{w}=0\right.$ and $\left.90 \%\right)$, taken under the illumination of a UV lamp.

the molecules become less congested, which leads to the formation of an $E$-isomer as it has a lower total electronic energy than a $Z$-isomer, as revealed by theoretical calculations (Fig. S11, ESI $\dagger$ ). However, in the case of (Z)-o-BPTPE, the $Z$-isomer is more stable than the $E$-isomer. Therefore, it is deduced that the spatial repulsion from $\phi_{3}$ should have functioned as a crucial driving force to bring about the parallel location of $\phi_{1}$ and $\phi_{2}$. Intramolecular $\pi-\pi$ stacking interactions and spatial congestion work collectively, resulting in the stereoselectivity of the reactions and the folded conformation of the products (Scheme S2, ESI $\dagger$ ).

Unlike most TPE derivatives that are almost non-fluorescent in a solution state, these folded TPE derivatives show good emissions in THF solutions. As an example, (Z)-o-BBPTPE shows an acceptable emission peak at $492 \mathrm{~nm}$. The fluorescence quantum yield $\left(\Phi_{\mathrm{F}}\right)$ is $45 \%$, measured in dilute THF solution, which is much higher than that of BBPTPE $(0.62 \%)$ (Table S1, ESI $\dagger)$. As revealed by the crystal structures of folded luminogens, the $\phi_{2} \mathrm{~s}$ in the TPE unit are located close to and in an approximately parallel pattern with the $\phi_{1} \mathrm{~s}$. As the rotations of the $\phi_{2} \mathrm{~s}$ are restricted greatly, the nonradiative decay of the excited state is thus reduced, leading to reasonably high emissions in solutions. These folded luminogens show better emissions in aggregates. Fig. 3A displays the photoluminescence (PL) spectra of (Z)-o-BBPTPE in THF-water mixtures as an example. It can be seen that the emission of (Z)-o-BBPTPE intensifies progressively without a significant change in the maximum absorption wavelength (Fig. S12, ESI $†$ ), with the addition of water. Since (Z)-o-BBPTPE is insoluble in water, its molecules should have aggregated in the aqueous medium, demonstrating that the enhanced emission is caused by the formation of aggregates. A slight hypsochromic shift is observed in high water fractions, which is attributed to the morphological change from amorphous to crystalline. ${ }^{8}$ The linear analogue BBPTPE, however, shows typical AIE characteristics (Fig. 3B). The film of (Z)-o-BBPTPE shows intense emission with a maximum at $472 \mathrm{~nm}$. The absolute $\Phi_{\mathrm{F}}$ of the film is $72 \%$, which is much higher than that in solution. This is because the motions of rotatable phenyl rings $\left(\phi_{3} \mathrm{~s}\right)$ are also suppressed by the spatial constraint in the aggregate state. The radiative relaxation of the excited state becomes dominant, affording improved emission efficiencies. Similar emission behaviors are also detected in other folded luminogens, revealing aggregation-enhanced emission (AEE) attributes.

The computational results from the analysis of (Z)-o-BPTPE verify that the phenyl rotors in the TPE unit are sensitive to various rotation barriers because of the folded conformation (Fig. S13, ESI $\dagger$ ). $\phi_{2}$ experiences a much higher rotation barrier than $\phi_{3}$, indicating that the rotational motions of $\phi_{2}$ are basically suppressed but $\phi_{3}$ is rotatable with little constraint. ${ }^{12}$ In other words, the intramolecular rotation of the TPE unit is restricted by $\sim 50 \%$ in the isolated state. The intramolecular rotation of $\phi_{3}$ can be further suppressed by spatial constraint in the condensed phase. These results strongly support that RIR is responsible for the AIE effect of most TPE derivatives.

In summary, a stereoselective synthesis of TPE derivatives with a folded $Z$-conformation is presented. The intramolecular $\pi-\pi$ stacking interaction and spatial congestion are responsible for the stereoselectivity of the reaction and the formation of the folded structure. These folded TPE derivatives show good fluorescence with much higher emission efficiencies than the linear TPE derivatives, owing to the partial suppression of the intramolecular rotation of phenyl rotors in the TPE unit. They possess AEE attributes and become more emissive in the solid state. These results reveal that internal control of the intramolecular rotations endows the luminogens with improved emissions in solution, suggesting that RIR is responsible for the AIE phenomenon. Further exploration of this folded system is in progress.

We acknowledge financial support from the National Natural Science Foundation of China (51273053), the Fundamental Research Funds for the Central Universities (2013ZZ0002), the Guangdong Innovative Research Team Program of China (20110C0105067115) and the Research Grants Council of Hong Kong (HKUST2/CRF/10).

\section{Notes and references}

1 J. Luo, Z. Xie, J. W. Y. Lam, L. Cheng, H. Chen, C. Qiu, H. S. Kwok, X. Zhan, Y. Liu, D. Zhu and B. Z. Tang, Chem. Commun., 2001, 1740. 2 (a) Z. Li, Y. Dong, B. Mi, Y. Tang, M. Häussler, H. Tong, Y. Dong, J. W. Y. Lam, Y. Ren, H. H. Y. Sun, K. S. Wong, P. Gao, I. D. Williams, H. S. Kwok and B. Z. Tang, J. Phys. Chem. B, 2005, 109, 10061; (b) Z. Zhao, Z. Wang, P. Lu, C. Y. K. Chan, D. Liu, J. W. Y. Lam, H. H. Y. Sung, I. D. Williams, Y. Ma and B. Z. Tang, Angew. Chem., Int. Ed., 2009, 48, 7608; (c) J. Huang, N. Sun, Y. Dong, R. Tang, P. Lu, P. Cai, Q. Li, D. Ma, J. Qin and Z. Li, Adv. Funct. Mater., 2013, 23, 2329.

3 (a) Y. Hong, J. W. Y. Lam and B. Z. Tang, Chem. Soc. Rev., 2011, 40, 5361; (b) Z. Zhao, J. W. Y. Lam and B. Z. Tang, Curr. Org. Chem., 2010, 14, 2109; (c) Z. Zhao, J. W. Y. Lam and B. Z. Tang, Soft Matter, 2013, 9, 4564.

4 (a) J. Shi, N. Chang, C. Li, J. Mei, C. Deng, X. Luo, Z. Liu, Z. Bo, Y. Dong and B. Z. Tang, Chem. Commun, 2012, 48, 10675; (b) H. Tong, Y. Dong, Y. Hong, M. Häussler, J. W. Y. Lam, H. H. Y. Sung, X. Yu, J. Sun, I. D. Williams, H. S. Kwok and B. Z. Tang, J. Phys. Chem. C, 2007, 111, 2287.

5 J. E. McMurry, Chem. Rev., 1989, 89, 1513.

6 (a) J. Zhou, Z. Chang, Y. Jiang, B. He, M. Du, P. Lu, Y. Hong, H. S. Kwok, A. Qin, H. Qiu, Z. Zhao and B. Z. Tang, Chem. Commun., 2013, 49, 2491; (b) Z. Zhao, S. Chen, J. W. Y. Lam, Z. Wang, P. Lu, F. Mahtab, H. H. Y. Sung, I. D. Williams, Y. Ma, H. S. Kwok and B. Z. Tang, J. Mater. Chem., 2011, 21, 7210.

7 M. Xue, L. Ding, L. Lin, Y. Lu, B. He, Y. Deng, Y. Guo, Y. Hong, J. W. Y. Lam, H. Qiu, Z. Zhao and B. Z. Tang, Asian J. Org. Chem., 2012, 1, 331.

8 Z. Zhao, J. W. Y. Lam, C. Y. K. Chan, S. Chen, J. Liu, P. Lu, M. Rodriguez, J.-L. Maldonado, G. Ramos-Ortiz, H. H. Y. Sung, I. D. Williams, H. Su, K. S. Wong, Y. Ma, H. S. Kwok, H. Qiu and B. Z. Tang, Adv. Mater., 2011, 23, 5430.

9 C. A. Hunter and J. K. M. Sanders, J. Am. Chem. Soc., 1990, 112, 5525.

10 S. M. Mathew, J. T. Engle, C. J. Ziegler and C. S. Hartley, J. Am. Chem. Soc., 2013, 135, 6714 .

11 J. L. Xia, S. H. Liu, F. Cozzi, M. Mancinelli and A. Mazzanti, Chem.-Eur. J., 2012, 18, 3611.

12 Q. Wu, Q. Peng, Y. Niu, X. Gao and Z. Shuai, J. Phys. Chem. A, 2012, 116, 3881. 